Series A

I. MATHEMATICA

375

\title{
ZUR THEORIE DER GEWÖHNLICHEN DIFFERENTIALGLEICHUNGEN
}

VON

ALEXANDER DINGHAS

Rolf Nevanlinna zum 70. Geburtstag

H E L S I N K I 1966

S U O M A L A I N E T IEDEAKATEMIA

https://doi.org/10.5186/aasfm.1966.375 
Am 14. Mai 1965 vorgelegt von P. J. Mrrberg und Olli Lehto 


\section{Zur Theorie der gewöhnlichen Differentialgleichungen}

1. Einleitung. Es bedeute zunächst $O_{x}$ eine offene (nicht leere) Teilmenge der reellen Geraden

$$
\boldsymbol{R}_{x}=\{x \mid-\infty<x<+\infty\}
$$

und

$$
f=\left\{f(x) \mid x \in O_{x}\right\}
$$

eine reellwertige stetige Abbildung von $O_{x}$ in $\boldsymbol{R}_{x}$. Man setze für $n=1,2, \ldots, \quad x \in O_{x}$ und hinreichend kleines $h>0$

$$
S_{h}^{n} f(x)=\frac{1}{(2 h)^{n}} \sum_{k=0}^{n}(-1)^{k}\left(\begin{array}{l}
n \\
k
\end{array}\right) f(x+(n-2 k) h) .
$$

Dann gilt, sofern $f$ an der Stelle $x$ eine $n$-te Ableitung $f^{(n)}(x)$ hat, die Gleichung ${ }^{1}$ )

$$
S^{n} f(x)=\lim _{h \rightarrow 0} S_{h}^{n} f(x)=f^{(n)}(x) .
$$

Im folgenden wird

$$
\underline{S}^{n} f(x)=\lim _{h \rightarrow 0} S_{h}^{n} f(x)
$$

und

$$
\bar{S}^{n} f(x)=\varlimsup_{h \rightarrow 0} S_{h}^{n} f(x)
$$

gesetzt und für $\underline{S}^{n} f(x)=\bar{S}^{n} f(x) \quad$ (im Einklang mit (1.2)) $\quad S^{n} f(x)$ geschrieben.

In der vorliegenden Arbeit wird zunächst die Frage untersucht, inwieweit die Ungleichungen

$$
\underline{S}^{n} f(x) \leqq g(x, f(x))
$$

und

\footnotetext{
1) Man vgl. etwa [7], S. 12.
} 


$$
\overline{S^{n}} f(x) \geqq g(x, f(x)),
$$

wobei $g(x, y)$ reellwertig und stetig in $O=O_{x} \times \boldsymbol{R}_{y}$ ist, die Existenz von $f^{(n)}(x)$ und das Bestehen der Differentialgleichung

$$
f^{(n)}(x)=g(x, f(x))
$$

zur Folge haben. Diese Frage kann hier nur für $n=1$ und $n=2$ behandelt werden. Eigentlich ist der grösste Teil der Arbeit dem Funktional

$$
D^{n}(J, f)=\frac{1}{|b-a|^{n}} \sum_{k=0}^{n}(-1)^{k}\left(\begin{array}{l}
n \\
k
\end{array}\right) f\left(\left(1-\frac{k}{n}\right) a+\frac{k}{n} b\right)
$$

und seinen Anwendungen auf die Theorie der gewöhnlichen Differentialgleichungen gewidmet, wobei $J=[a, b]$ ein (beliebiges) Intervall von $O_{x}$ bedeutet. Diese Intervallfunktion, obwohl für $n>1$ nicht additiv, gestattet, bei geeigneter Zerlegung von $J$ in $n+1$ kongruente, nicht unbedingt punktfremde Teilintervalle $J_{0}, \ldots, J_{n}$, eine Darstellung von der Form

$$
D^{n}(J, f)=\sum_{k=0}^{n} \mu_{k}(n) D^{n}\left(J_{k}, f\right)
$$

mit $0<\mu_{k}(n)<1(k=0,1, \ldots, n), \mu_{0}(n)+\ldots+\mu_{n}(n)=1$ und somit die Bildung von Maximalketten, die für das Goursat-Verfahren charakteristisch sind.

2. Die Fälle $n=1$ und $n=2$. Nachfolgende Sätze können einfach bewiesen werden:

Satz 1. Es sei $g(x, y)$ stetig in $O=O_{x} \times \boldsymbol{R}_{y}$ und $f$ stetig in $O_{x}$. Gilt dann

$$
\bar{S}^{1} f(x) \leqq g(x, f(x))
$$

und

$$
\underline{S}^{1} f(x) \geqq g(x, f(x))
$$

in jedem Punkt von $O_{x}$, so ist $f$ differenzierbar in $O_{x}$ und

$$
f^{\prime}(x)=g(x, f(x)) .
$$

Aus dem Satz 1 folgt, wenn man dort $g=0$ voraussetzt, der Satz:

Satz 2. Ist $f$ in einem offenen Intervall $J_{0}$ von $\boldsymbol{R}_{\boldsymbol{x}}$ stetig und gilt dort

$$
\bar{S}^{1} f(x) \leqq 0
$$


und

$$
\underline{S}^{1} f(x) \geqq 0
$$

so ist $f$ eine Konstante.

Satz 3. Es sei $f$ stetig in $O_{x}, g(x, y)$ stetig in $O$ und

$$
\begin{aligned}
& \frac{S}{2}^{2} f(x) \leqq g\left(x, f^{\prime}(x)\right), \\
& \bar{S}^{2} f(x) \geqq g(x, f(x))
\end{aligned}
$$

in jedem Punkt von $O_{x}$. Dann ist $f(x)$ zweimal steitig differenzierbar und genügt der Differentialgleichung

$$
f^{\prime \prime}(x)=g(x, f(x)) .
$$

Nimmt man hier $g=0$, so erhält man den Satz:

Satz 4. Ist $f$ in einem offenen Intervall $J_{0}$ von $\boldsymbol{R}_{x}$ stetig und gilt dort

$$
\underline{S}^{2} f(x) \leqq 0
$$

und

$$
\bar{S}^{2} f(x) \geqq 0,
$$

so ist $f$ eine lineare Funktion.

Ersetzt man hier die Ungleichungen (2.8) und (2.9) durch die Gleichung $S^{2} f(x)=0 \quad\left(x \in J_{0}\right)$, so erhält man ein klassisches Resultat von H. A. Schwarz ${ }^{2}$ ).

Wir beweisen zunächst den Satz 2 .

Es sei $J=[a, b]$ ein abgeschlossenes Teilintervall von $J_{0}$. Man setze

$$
h(x)=f(x)-f(a)+\varepsilon(x-a)+\varepsilon_{1} \quad\left(\varepsilon, \varepsilon_{1}>0\right) .
$$

Dann ist wegen (2.4) und (2.5)

$$
S^{1} h(x)=\varepsilon>0 \quad(x \in J) .
$$

Wäre nun $H=\{x \mid x \in J, h(x)<0\}$ nicht leer, so müsste der Punkt $x_{0}=\inf \{x \mid x \in H\}$ in $] a, b[$ liegen. Andererseits müsste mit Rücksicht auf (2.11) die Ungleichung $h\left(x_{0}+\eta\right)>h\left(x_{0}-\eta\right)$ für alle hinreichend kleinen $\eta>0$ gelten. Das widerspricht jedoch der Definition von $x_{0}$. Somit gilt $h \geqq 0$ in $J$. Ähnlich zeigt man, dass die Funktion

$$
f(x)-f(a)-\varepsilon(x--a)-\varepsilon_{1}
$$

2) Man vgl. [1], S. 81, [9], S. 258 ff., und [10], S. 341 ff. 
in $J$ nicht positiv sein kann. Durch Grenzübergang $\varepsilon \rightarrow 0$ und $\varepsilon_{1} \rightarrow 0$ erhält man nun $f(x)=f(a)$ in $J$ und durch (monotone) Ausschöpfung von $J_{0}$ durch abgeschlossene Intervalle $f(x)=f(a)$ in $J_{0}$.

Zum Beweis des Satzes 4 bilde man mit Schwarz ${ }^{3}$ ) die Funktionen

$$
\begin{aligned}
& h_{2}(x)=f(x)-L f(x)-\frac{\varepsilon}{2}(b-x)(x-a), \\
& h_{1}(x)=f(x)-L f(x)+\frac{\varepsilon}{2}(b-x)(x-a),
\end{aligned}
$$

mit festen $a, b \in J_{0} \quad(a<b)$, und

$$
L f(x)=\frac{b-x}{b-a} f(a)+\frac{x-a}{b-a} f(b) .
$$

Dann wird wegen (2.8) und (2.9)

$$
\underline{S}^{2} h_{1}(x)=\underline{S}^{2} f(x)-\varepsilon \leqq-\varepsilon<0
$$

und

$$
{\overline{S^{2}}}^{2}(x)=\overline{S^{2}} f(x)+\varepsilon \geqq \varepsilon>0 .
$$

Es sei jetzt

$$
M=\sup \left\{h_{2}(x) \mid x \in[a, b]\right\}
$$

und

$$
E_{a b}=\left\{x \mid x \in[a, b], h_{2}(x)=M\right\} .
$$

Dann hat $E_{a b}$ die Eigenschaften:

1. $E_{a b} \neq \varnothing$. (Nach dem Satz von Weierstrass, wonach jede in einem abgeschlossenen Intervall stetige Funktion ein Maximum hat.)

2. $\left.E_{a b} \cap\right] a, b\left[=\varnothing\right.$. Gäbe es nämlich einen Punkt $x_{0}$ von $E_{a b}$ in $] a, b[$, so müsste dieser Häufungspunkt von links und von rechts von Punkten von $E_{a b}$ sein. Eine leichte Überlegung zeigt dann, dass (2.14) nicht gelten kann.

Somit enthält $E_{a b}$ höchstens die Punkte $a, b$. Wegen $h_{2}(a)=$ $h_{2}(b)=0$ gilt also $h_{2}(x) \leqq 0$ in $[a, b]$. Ein entsprechender Schluss liefert die Ungleichung $h_{1}(x) \geqq 0$ in $[a, b]$. Lässt man nun $\varepsilon \rightarrow 0$ konvergieren, so erhält man $f(x) \leqq L f(x)$ und $f(x) \geqq L f(x)$, d. h. $f(x)=L f(x)$. Mithin ist $f(x)$ eine lineare Funktion in jedem Intervall $[a, b]$ von $J_{0}$, d. h. auch in $J_{0}$.

3) [10], S. $341 \mathrm{ff}$. 
3. Beweis der Sätze 1 und 3. Verallgemeinerungen. Es sei $x_{0} \in O_{x}$ und $J_{x_{0}}$ ein offenes Intervall in $O_{x}$ mit dem Mittelpunkt $x_{0}$, derart dass die Differentialgleichung

$$
y^{\prime}=g(x, y)
$$

in $J_{x_{0}}$ eine Lösung $y=y(x)$ hat. Wir bilden die Funktion $q=q(x)$,

$$
q(x)=\int_{x_{0}}^{x}\{g(t, f(t))-g(t, y(t))\} d t,
$$

und beachten, dass

$$
S^{1} q(x)=g(x, f(x))-g(x, y(x))
$$

gilt. Andererseits ist wegen (2.1), (2.2) und

d. h.

$$
\begin{aligned}
& S^{1} y(x)=y^{\prime}(x)=g(x, y(x)), \\
& \bar{S}^{1}(f(x)-y(x)) \leqq g(x, f(x))-g(x, y(x)), \\
& \underline{S}^{1}(f(x)-y(x)) \geqq g(x, f(x))-g(x, y(x)),
\end{aligned}
$$

und

$$
\bar{S}^{1}(f(x)-y(x)-q(x)) \leqq 0
$$

Somit ist (nach dem Satz 2) $f-y-q$ eine Konstante, d. h. (wegen $f=y+q+$ Konstante) $f$ differenzierbar. Das beweist den Satz 1 .

Der Satz 3 lässt sich analog beweisen. Wir setzen hier (unter Beibehaltung der Bedeutung von $J_{x_{0}}$ )

$$
p(x)=\int_{x_{0}}^{x}(x-t)\{g(t, f(t))-g(t, y(t))\} d t,
$$

wobei $y$ irgendeine (fest gewählte) Lösung der Differentialgleichung

$$
y^{\prime \prime}=g(x, y)
$$

in $J_{x_{0}}$ bedeutet, die durch $x_{0}$ geht. Wegen

$$
p^{\prime \prime}(x)=g(x, f(x))-g(x, y(x))
$$

folgt, mit Rücksicht auf (2.6),

$$
\underline{S}^{2}(f(x)-y(x)-p(x)) \leqq 0
$$

und 


$$
\overline{S^{2}}(f(x)-y(x)-p(x)) \geqq 0 .
$$

Somit hat $f-y-p$, nach dem Satz 4, die Form $A x+B$ mit Konstanten $A, B$ in $J_{x_{0}}$, und es gilt $f(x)=y(x)+p(x)+A x+B$ in $J_{x_{\theta}}$. Das beweist die zweimalige Differenzierbarkeit von $f$ und zugleich den Satz 3.

Die Sätze 1-4 können dahin verallgemeinert werden, dass man die Differentialgleichungen (3.1) und (3.8) durch entsprechende Systeme ersetzt. Im folgenden ersetzen wir diese Gleichungen durch

$$
\mathfrak{y}^{\prime}=\mathfrak{g}(x, \mathfrak{y})
$$

und

$$
\mathfrak{y}^{\prime \prime}=\mathfrak{g}(x, \mathfrak{y})
$$

und fassen $\mathfrak{y}$ bzw. $\mathfrak{g}$ als Vektoren des $n$-dimensionalen euklidischen Raumes $\boldsymbol{E}^{n}$ auf. Ist $\mathfrak{y}=\left(y_{1}, \ldots, y_{n}\right)$ ein Punkt von $\boldsymbol{E}^{n}$, so soll (3.11) bzw. (3.12) das Bestehen der Differentialgleichungssysteme

$$
y_{k}^{\prime}=g_{k}\left(x, y_{1}, \ldots, y_{n}\right) \quad(k=1, \ldots, n)
$$

bzw.

$$
y_{k}^{\prime \prime}=g_{k}\left(x, y_{1}, \ldots, y_{n}\right)
$$

zum Ausdruck bringen.

Satz 5. Die Funktion $\mathfrak{g}(x, \mathfrak{y})$ sei in $J \times \boldsymbol{E}^{n}$, wobei $J$ ein offenes, nicht leeres Intervall von $\boldsymbol{R}_{\boldsymbol{x}}$ ist, definiert und stetig. Genügt dann die stetige Funktion $\mathfrak{f}=\left(f_{1}, \ldots, f_{n}\right)$ den Bedingungen:

1) $\mathfrak{f} \in E^{n}$,

2) $\quad \bar{S}^{1} f_{k}(x) \leqq g_{k}\left(x, f_{1}(x), \ldots, f_{n}(x)\right)$

und

3) $\quad \underline{S}^{1} f_{k}(x) \geqq g_{k}\left(x, f_{1}(x), \ldots, f_{n}(x)\right)$

für alle $k=1, \ldots, n$ und alle $x \in J$, so ist $\mathfrak{f}$ differenzierbar und genügt der Differentialgleichung (3.11).

Satz 6. Gilt (unter denselben Voraussetzungen für $\mathfrak{f}, \mathfrak{g}$ ) für $x \in J$

$$
\underline{S}^{2} f_{k}(x) \leqq g_{k}\left(x, f_{1}(x), \ldots, f_{n}(x)\right)
$$

und

$$
\bar{S}^{2} f_{k}(x) \geqq g_{k}\left(x, f_{1}(x), \ldots, f_{n}(x)\right)
$$


anstelle von 2) und 3), so ist $\mathfrak{f}$ zweimal stetig differenzierbar in $J$, und es gilt

$$
\mathfrak{f}^{\prime \prime}(x)=\mathfrak{g}(x, \mathfrak{f}(x))
$$

in $J$.

Der Beweis dieser beiden Sätze läuft wie im Falle $n=1$ und wird dem Leser überlassen.

Aus den Sätzen 1 und 3 folgen, wenn man dort die Funktion $g(x, y)$ unabhängig von $y$ voraussetzt, die Sätze:

Satz 7. Die reellwertige Funktion $f$ sei in $O \subseteq \boldsymbol{R}_{\boldsymbol{x}}$ stetig. Existiert dann $S^{1} f(x)$ in $O$ und ist dort stetig, so hat $f(x)$ eine Ableitung $f^{\prime}(x)$ in $O$, und es gilt $f^{\prime}(x)=S^{1} f(x)$.

Satz 8. Die reellwertige Funktion $f$ sei in $O \subseteq \boldsymbol{R}_{x}$ stetig. Existiert dann $S^{2} f(x)$ in $O$ und ist dort stetig, so hat $f(x)$ eine zweite Ableitung $f^{\prime \prime}(x)$ gleich $S^{2} f(x)$ in jedem Punkt von $O$.

Es sei in der Tat $x_{0}$ ein Punkt von $O$ und $J_{x_{0}}$ ein offenes Intervall um $x_{0}$ in $O$. Man setze

$$
f^{*}(x)=\int_{x_{0}}^{x} S^{1} f(t) d t \quad\left(x \in J_{x_{0}}\right) .
$$

Dann ist $f^{*^{\prime}}(x)=S^{1} f(x)$, also auch $S^{1} f^{*}(x)=S^{1} f(x)$. Somit ist $f^{*}(x)-f(x)$ eine Konstante. Das beweist den Satz 7 .

Definiert man $f^{*}(x)$ durch die Gleichung

$$
f^{*}(x)=\int_{x_{0}}^{x}(x-t) S^{2} f(t) d t \quad\left(x \in J_{x_{0}}\right),
$$

so erhält man, sofern $f$ ein stetiges $S^{2} f(x)$ in $J_{x_{0}}$ hat, die Gleichung

d. h.

$$
f^{* \prime \prime}(x)=S^{2} f^{*}(x)=S^{2} f(x) \quad\left(x \in J_{x_{0}}\right),
$$

$$
S^{2}\left(f(x)-f^{*}(x)\right)=0 \quad \text { in } \quad J_{x_{0}} .
$$

Somit ist $f(x)-f^{*}(x)$ linear in $J_{x_{0}}$ und somit $f$ dort zweimal differenzierbar. Das beweist den Satz $8^{4}$ ).

4. Heranziehung des Funktionals $D^{n}(J, f)$. Zwischen den Funktionalen $S_{h}^{n} f(x)$ und $D^{n}(J, f)$ besteht ausser der Tatsache, dass beim ersteren Funktional der Mittelpunkt $x$ bei Grenzübergängen fest

4) Man vgl. in diesem Zusammenhang auch die Arbeiten von R. D. James, etwa [5]. Einen Einblick in diese Arbeit konnte ich allerdings nur über das Buch von R. L. Jeffery [6] erhalten. 
bleibt, strukturell kein Unterschied. Vielmehr liegen die Vorteile des zweiten Funktionals in der $n$-Additivität (1.9) desselben, die, wie bereits erwähnt, die Anwendbarkeit des Goursatschen Verfahrens ermöglicht.

Es bezeichne $X$ eine nicht leere, konvexe Teilmenge eines (reellen oder komplexen) Banach-Raumes $\boldsymbol{B}_{0}$, [3]. Es seien ferner $a_{\mathbf{0}}, b_{0}, x \in \boldsymbol{B}_{\mathbf{0}}$, $a_{0} \neq b_{0}$ und

$$
I_{0}=\left\{x \mid x=(1-\lambda) a_{0}+\lambda b_{0}, 0 \leqq \lambda \leqq 1\right\}
$$

ein festes (Ausgangs-) Intervall von $X$. Wir verstehen unter $J=$ $[a, b]$ ein (beliebiges) Teilintervall von $I_{0}$ mit dem Anfangspunkt $a=\left(1-\lambda^{\prime}\right) a_{0}+\lambda^{\prime} b_{0}$ und dem Endpunkt $b=\left(1-\lambda^{\prime \prime}\right) a_{0}+\lambda^{\prime \prime} b_{0} \quad(0 \leqq$ $\left.\lambda^{\prime}<\lambda^{\prime \prime} \leqq 1\right)$ und definieren das in 1 eingeführte Funktional $D^{n}(J, f)$ folgendermassen:

Definition 1. Es sei $f: I_{0} \rightarrow \boldsymbol{B}$ eine stetige Abbildung von $I_{0}$ in einen Banach-Raum B. Dann soll der Ausdruck

$$
D^{n}(J, f)=\frac{1}{\|J\|^{n}} \sum_{k=0}^{n}(-1)^{k}\left(\begin{array}{l}
n \\
k
\end{array}\right) f\left(\left(1-\frac{k}{n}\right) a+\frac{k}{n} b\right)
$$

die $n$-te Differenz von $f$ auf $J$ heissen. Dabei ist $\|J\|=\|b-a\|$ die Norm von $b-a$.

Ist $\left\|a_{0}-b_{0}\right\|=1 \quad$ und $a=\left(1-\lambda_{a}\right) a_{0}+\lambda_{a} b_{0}, \quad b=\left(1-\lambda_{b}\right) a_{0}+\lambda_{b} b_{0}$ $\left(0 \leqq \lambda_{a}<\lambda_{b} \leqq 1\right)$, so ist $\|\boldsymbol{J}\|=\lambda_{b}-\lambda_{a}$. Für ein Intervall $J_{0}$ von $\boldsymbol{R}$ und somit reellen $a_{0}, b_{0}$ wird $D^{n}(J, f)$ durch die Gleichung

$$
D^{n}(J, f)=\frac{1}{(b-a)^{n}} \sum_{k=0}^{n}(-1)^{k}\left(\begin{array}{l}
n \\
k
\end{array}\right) f\left(\left(1-\frac{k}{n}\right) a+\frac{k}{n} b\right)
$$

gegeben.

Definition 2. Es sei $N$ eine (abzählbare) Basis des numerischen Intervalls $\Lambda=[0,1]$. Dann soll $\mathfrak{N}(N)$ die Basis von $I_{0}$ bedeuten, deren Punkte $x$ durch $(1-\lambda) a_{0}+\lambda b_{0} \quad(\lambda \in N)$ gegeben werden.

Definition 3. Unter $\left\{J_{x}\right\}$ wird im folgenden, bei gegebenem $x$ in $I_{0}$, die Gesamtheit aller (geordneten) Teilintervalle $J_{x}$ mit Endpunkten in $I_{0}$ verstanden, die den Punkt $x$ enthalten.

Definition 4. Gibt es ein Element $D^{n} f(x)$ von $\boldsymbol{B}$ derart, dass

$$
\lim _{\left\|J_{x}\right\| \rightarrow 0}\left\|D^{n} f(x)-(-n)^{n} D^{n}\left(J_{x}, f\right)\right\|=0
$$

gilt, so soll $D^{n} f(x)$ die $n$-te Intervall-Derivierte auf $I_{0}$ von $f$ in $x$ heissen.

Ist $\boldsymbol{B}_{0}=\boldsymbol{B}=\boldsymbol{R}$, also $f$ eine reellwertige, stetige Abbildung von $I_{0}$ in $\boldsymbol{R}$, so kann man neben $D^{n} f(x)$ eine obere und eine untere $n$-te Intervall-Derivierte definieren. 
Definition 5. Ist $f$ eine stetige Abbildung von $I_{0} \subset \boldsymbol{R}$ in $\boldsymbol{R}$ und $x \in I_{0}$, so sollen die Grössen

$$
\underline{D}^{n} f(x)=\lim _{\| \overline{J_{x} \| \rightarrow 0}}(-n)^{n} D^{n}\left(J_{x}, f\right)
$$

und

$$
\bar{D}^{n} f(x)=\varlimsup_{\left\|J_{x}\right\| \rightarrow 0}(-n)^{n} D^{n}\left(J_{x}, f\right)
$$

entsprechend die obere bzw. die untere $n$-te Intervall-Derivierte von $f$ in $x$ heissen ${ }^{5}$.

5. Eine notwendige und hinreichende Bedingung für Polynom-Abbildungen. Wir betrachten zunächst das Intervall $J_{0}=[0,1]$ und nehmen als Basis die Menge $N_{0}$ aller dyadischen Brüche in $J_{0}$, d. h. alle rationalen Zahlen von der Form

$$
r=\frac{a_{1}}{2}+\frac{a_{2}}{2^{2}}+\ldots+\frac{a_{k}}{2^{k}}
$$

mit $a_{r}=0$ oder $=1$. Im folgenden werden zur Abschätzung der Grössen $\underline{D}^{n} f(x), \quad \bar{D}^{n} f(x)$ und $D^{n} f(x)$ lediglich Intervalle $J_{x}$ verwendet werden, deren Endpunkte $a, b$ dyadische Brüche sind.

Hilfssatz 1. Es bedeute $f$ eine stetige Abbildung von $J=[0,1]$ in $\boldsymbol{R}$ mit der Eigenschaft $D^{n} f(x)=0 \quad(x \in J)$. Dann ist $f$ ein Polynom höchstens vom Grade $n-1$.

Allgemeiner gilt der Satz:

Hilfssatz 2. Es bedeute $I_{0}$ das Intervall (4.1) der konvexen Teilmenge $X$ des Banach-Raumes $\boldsymbol{B}_{0}$ und $f: I_{0} \rightarrow \boldsymbol{B}$ eine stetige Abbildung von $I_{0}$ in einen Banach-Raum $\boldsymbol{B}$. Gilt dann $D^{n} f(x)=0$ in jedem Punkt $x=(1-\lambda) a_{0}+\lambda b_{0} \quad(0 \leqq \lambda \leqq 1)$ von $I_{0}$, so hat $f$ die Form

$$
1 f_{0}+\lambda f_{1}+\ldots+\lambda^{n-1} f_{n-1}
$$

mit $f_{0}, f_{1}, \ldots, f_{n-1}$ aus $\boldsymbol{B}$.

Da für $f=f(x), \quad x=x(\lambda)=(1-\lambda) a_{0}+\lambda b_{0}$ und $f(x(\lambda))=1 f_{0}+$ $\lambda f_{1}+\ldots+\lambda^{n-1} f_{n-1}\left(f_{0}, f_{1}, \ldots, f_{n-1}\right.$ fest in $\left.\boldsymbol{B}\right)$ stets $D^{n} f(x)=0$ gilt, ist die Bedingung $D^{n} f(x)=0$ notwendig und hinreichend dafür,

$\left.{ }^{5}\right)$ Der Faktor $(-n)^{n}$ fällt fort, wenn man die Summe rechts in (4.3) durch $(a-b)^{n} / n^{n}$ statt durch $(b-a)^{n}$ dividiert. Diese Definition, d. h. die Heranziehung des Funktionals $\Delta^{n}(J, f)=(-n)^{n} D^{n}(J, f)$, entspricht mehr der Definition von $S_{h}^{n} f(x)$. In den nachfolgenden Entwicklungen wird das Funktional $D^{n}(J, f)$ lediglich aus technischen Gründen vorgezogen. 
dass $f$ auf $I_{0}$ ein Polynom in $\lambda$ mit Koeffizienten aus $\boldsymbol{B}$ ist. Entsprechendes gilt für den numerischen Fall des Hilfssatzes 1.

Zum Beweis des Hilfssatzes 1 bilden wir neben der Grösse

$$
F(J)=\sum_{k=0}^{n}(-1)^{k}\left(\begin{array}{l}
n \\
k
\end{array}\right) f\left(\frac{k}{n}\right)
$$

die Grössen

$$
F\left(J_{k}\right)=\sum_{l=0}^{n}(-1)^{l}\left(\begin{array}{l}
n \\
l
\end{array}\right) f\left(\frac{k+l}{2 n}\right)
$$

mit

$$
J_{k}=\left[\frac{k}{2 n}, \frac{k}{2 n}+\frac{1}{2}\right] \quad(k=0,1, \ldots, n)
$$

und beachten, dass dann

$$
D^{n}\left(J_{k}, f\right)=\frac{F\left(J_{k}\right)}{\left|J_{k}\right|^{n}}=2^{n} \frac{F\left(J_{k}\right)}{|J|^{n}} .
$$

Nun ist, wenn $q=k+l$ gesetzt wird,

$$
\sum_{k=0}^{n}\left(\begin{array}{l}
n \\
k
\end{array}\right) F\left(J_{k}\right)=\sum_{q=0}^{2 n}\left\{\sum_{l=0}^{n}(-1)^{l}\left(\begin{array}{c}
n \\
q-l
\end{array}\right)\left(\begin{array}{l}
n \\
l
\end{array}\right) f\left(\frac{q}{2 n}\right)\right\}
$$

und somit mit Rücksicht auf die bekannte Identität

der Kombinatorik ${ }^{6}$ )

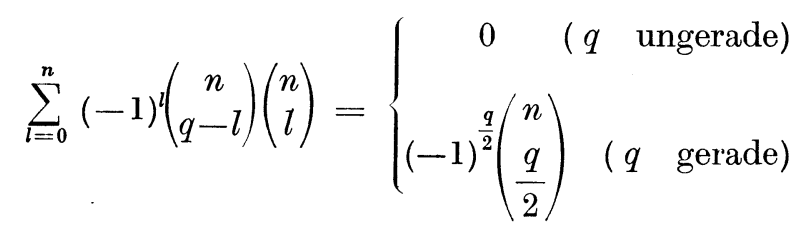

$$
\sum_{k=0}^{n}\left(\begin{array}{l}
n \\
k
\end{array}\right) F\left(J_{k}\right)=\sum_{k=0}^{n}(-1)^{k}\left(\begin{array}{l}
n \\
k
\end{array}\right) f\left(\frac{k}{n}\right)=F(J) .
$$

Es ist also

$$
D^{n}(J, f)=\sum_{k=0}^{n} \mu_{k}(n) D^{n}\left(J_{k}, f\right)
$$

mit

$$
\mu_{k}(n)=\frac{1}{2^{n}}\left(\begin{array}{l}
n \\
k
\end{array}\right) \quad(k=0,1, \ldots, n)
$$

6) Man vgl. [2] sowie auch [8], S. 256. 
Hat man anstelle von $J=[0,1]$ das Intervall $I_{0}$, so ist mit $I=[a, b] \subseteq I_{0}$ und

$$
D^{n}(I, f)=\frac{1}{\|I\|^{n}} \sum_{k=0}^{n}(-1)^{k}\left(\begin{array}{l}
n \\
k
\end{array}\right) f\left(\left(1-\frac{k}{n}\right) a+\frac{k}{n} b\right)
$$

sowie

$$
\begin{gathered}
D^{n}\left(I_{k}, f\right)=\frac{1}{\left\|I_{k}\right\|^{n}} \sum_{l=0}^{n}(-1)^{l}\left(\begin{array}{l}
n \\
l
\end{array}\right) f\left(a+\frac{k+l}{2 n}(b-a)\right), \\
D^{n}(I, f)=\sum_{k=0}^{n} \mu_{k}(n) D^{n}\left(I_{k}, f\right) .
\end{gathered}
$$

Aus (5.5) und (5.7) erhält man nun wegen $\mu_{0}(n)+\ldots+\mu_{n}(n)=1$ die Ungleichungen

$$
\left|D^{n}(J, f)\right| \leqq \max _{0 \leqq k \leqq n}\left|D^{n}\left(J_{k}, f\right)\right|
$$

und

$$
\left\|D^{n}(I, f)\right\| \leqq \max \left\|D^{n}\left(I_{k}, f\right)\right\| .
$$

Liefert $f$ eine Abbildung von $J=[0,1]$ in $\boldsymbol{R}$, so findet man entsprechend

$$
\min _{0 \leqq k \leqq n} D^{n}\left(J_{k}, f\right) \leqq D^{n}(J, f) \leqq \max _{0 \leqq k \leqq n} D^{n}\left(J_{k}, f\right) .
$$

Mit Hilfe von (5.5) kann nun gezeigt werden, dass $F(J)$ gleich Null sein muss, falls $D^{n} f(x)$ (unter Zugrundelegung von $N_{0}$ ) in jedem Punkt $x$ von $J=[0,1]$ verschwindet.

Es bezeichne in der Tat $J_{1}^{\prime}$ ein Intervall aus der Menge $\left\{J_{0}\right.$, $\left.J_{1}, \ldots, J_{n}\right\}$, für das

$$
D^{n}\left(J_{1}^{\prime}, f\right)=\max _{0 \leqq k \leqq n} D^{n}\left(J_{k}, f\right)
$$

ist. Wir nehmen dieses Intervall zum Ausgangsintervall und bilden die Kette $J_{1}^{\prime}, J_{2}^{\prime}, \ldots$ (kurz: $\left.\left(J_{\sigma}^{\prime}\right)\right)$ durch folgendes Verfahren:

Jedes $J_{\sigma}^{\prime}(\sigma \geqq 2)$ gehört der Unterteilungsmenge von $J_{\sigma-1}^{\prime}$ in kongruente Teilintervalle nach dem Vorgang $J \rightarrow\left\{J_{0}, \ldots, J_{n}\right\}$ an und genügt der entsprechenden Bedingung (5.11). Danach gilt stets $J_{\sigma+1}^{\prime} \subseteq$ $J_{\sigma}^{\prime} \quad(\sigma=1,2, \ldots), \quad 2\left|J_{\sigma+1}^{\prime}\right|=\left|J_{\sigma}^{\prime}\right| \quad$ und

$$
D^{n}\left(J_{\sigma}^{\prime}, f\right) \leqq D^{n}\left(J_{\sigma+1}^{\prime}, f\right) \quad(\sigma=1,2, \ldots) .
$$

Man setze jetzt $x_{0}^{\prime}=\bigcap_{\sigma=1}^{\infty} J_{\sigma}^{\prime}$. Dann ist $x_{0}^{\prime} \in J$, und es gilt

$$
D^{n}(J, f) \leqq \lim _{\sigma \rightarrow+\infty} D^{n}\left(J_{\sigma}^{\prime}, f\right)=0 \text {. }
$$


Ersetzt man in (5.11) max durch min, so erhält man eine Folge $\left(J_{\sigma}^{\prime \prime}\right)$ von ineinandergeschachtelten Intervallen mit

$$
D^{n}\left(J_{\sigma}^{\prime \prime}, f\right) \geqq D^{n}\left(J_{\sigma+1}^{\prime \prime}, f\right)
$$

anstelle von (5.12). Wird dann noch $x_{0}^{\prime \prime}=\bigcap_{\sigma=1}^{\infty} J_{\sigma}^{\prime \prime}$ gesetzt, so wird

$$
D^{n}(J, f) \geqq \lim _{\sigma \rightarrow+\infty} D^{n}\left(J_{\sigma}^{\prime \prime}, f\right)=0
$$

und somit auch $D^{n}(J, f)=0$.

Durch ein entsprechendes Verfahren, das zu den Ungleichungen

$$
\left\|D^{n}(I, f)\right\| \leqq \lim _{\sigma \rightarrow \infty}\left\|D^{n}\left(I_{\sigma}^{\prime}, f\right)\right\|=n^{-n}\left\|D^{n} f\left(x_{0}^{\prime}\right)\right\|=0
$$

(bei entsprechender Definition und Bedeutung der Intervalle $I_{\sigma}^{\prime}$ ) führt, erhält man folgendes Teilergebnis in Zusammenhang mit dem Beweis des Hilfssatzes 1:

Gilt $D^{n} f(x)=0$ in jedem Punkt von $I$, so gilt $F(I)=0$. Dabei ist $I$ ein beliebiges Teilintervall von $I_{0}$.

6. Ende des Beweises der Hilfssätze 1 und 2. Der Beweis des Hilfssatzes 1 lässt sich nun folgendermassen zu Ende führen: Man gehe von den Gleichungen

$$
F\left(J_{k}\right)=\sum_{l=0}^{n}(-1)^{l}\left(\begin{array}{l}
n \\
l
\end{array}\right) f\left(\frac{k+l}{2 n}\right)=0 \quad(k=0,1,2, \ldots, n)
$$

aus und bilde die Summe

$$
\sum_{k=0}^{n}(-1)^{k} \alpha_{k} F\left(J_{k}\right)=0
$$

mit zunächst willkürlichen reellen $\alpha_{k}$. Daraus folgt, wenn man

$$
\gamma_{s}=\sum_{k=0}^{n}\left(\begin{array}{c}
n \\
s-k
\end{array}\right) \alpha_{k}
$$

setzt,

$$
\sum_{s=0}^{2 n}(-1)^{s} f\left(\frac{s}{2 n}\right) \gamma_{s}=0
$$

Man betrachte jetzt die Punkte

$$
x_{k}=\frac{k}{n} \quad(k=0,1, \ldots, n-1)
$$

von $J$, setze 


$$
\psi_{k}(x)=\prod_{\nu \neq k}\left(x-x_{\nu}\right) \quad(k=0,1, \ldots, n-1)
$$

und bilde das Polynom

$$
P_{n-1}(x)=\sum_{k=0}^{n-1} \frac{\psi_{k}(x)}{\psi_{k}\left(x_{k}\right)} f\left(\frac{k}{n}\right) .
$$

Ist dann

$$
\bar{f}(x)=f(x)-P_{n-1}(x) \quad(x \in J),
$$

so gilt offenbar, wegen $P_{n-1}^{(n)}(x) \equiv 0$ in $J$,

$$
\bar{F}(J)=\bar{F}\left(J_{1}\right)=\ldots=\bar{F}\left(J_{n}\right)=0,
$$

sofern $\bar{F}(J), \bar{F}\left(J_{1}\right), \ldots, \bar{F}\left(J_{n}\right)$ die mit Hilfe von $\bar{f}$ gebildeten Grössen $F(J), F\left(J_{1}\right), \ldots, F\left(J_{n}\right)$ bedeuten.

Nun folgt aus $\bar{F}(J)=0$ und

$$
\begin{aligned}
\bar{f}\left(\frac{k}{n}\right)=0 & (k=0,1,2, \ldots, n-1), \\
\bar{f}\left(\frac{s}{2 n}\right)=0 & (s=0,2,4, \ldots, 2 n),
\end{aligned}
$$

und somit gilt

$$
\sum_{s=1}^{n} \bar{f}\left(\frac{2 s-1}{2 n}\right) \gamma_{2 s-1}=0 .
$$

Es soll: nun gezeigt werden, dass man durch geeignete Wahl der $\alpha_{k}$ sämtliche Werte $\bar{f}((2 s-1) /(2 n))$ zum Verschwinden bringen kann.

Hilfssatz 3. Die Determinante des Gleichungssystems

$$
\sum_{k=0}^{n-1}\left(\begin{array}{c}
n \\
2 s-k-1
\end{array}\right) \alpha_{k}=0 \quad(s=1,2, \ldots, n)
$$

ist von Null verschieden.

Der Beweis dieses Hilfssatzes stützt sich weitgehend auf folgendes berühmte Kriterium von Hurwitz ${ }^{7}$ ) über Polynome, deren Nullstellen einen negativen Realteil besitzen:

Es sei

$$
P(x)=a_{0} x^{n}+a_{1} x^{n-1}+\ldots+a_{n} \quad\left(a_{0}=1, a_{n} \neq 0\right)
$$

ein Polynom $n$-ten Grades mit reellen Koeffizienten. Man setze $\Delta_{1}=a_{1}$ und definiere für $\lambda=2,3, \ldots, n$ die Determinanten $\Delta_{\lambda}$ durch die Gleichung

7) Man vgl. [4] und [11], S. 335 ff. 


$$
\Delta_{\lambda}=\left|\begin{array}{ccccccc}
a_{1} & a_{0} & 0 & 0 & 0 & \ldots & 0 \\
a_{3} & a_{2} & a_{1} & a_{0} & 0 & \ldots & 0 \\
a_{5} & a_{4} & a_{3} & a_{2} & a_{1} & \ldots & 0 \\
\cdot & & & & & & \\
\cdot & & & & & & \\
\cdot & & & & & & \\
a_{2 \lambda-1} & a_{2 \lambda-2} & a_{2 \lambda-3} & a_{2 \lambda-4} & a_{2 \lambda-5} & \ldots & a_{\lambda}
\end{array}\right| .
$$

Dann haben sämtliche Wurzeln von (6.8) dann und nur dann negativen Realteil, wenn alle Determinanten $\Delta_{1}, \Delta_{2}, \ldots, \Delta_{n}$ positiv sind.

Ausführlich geschrieben lauten die Determinanten $\Delta_{1}, \Delta_{2}, \Delta_{3}, \ldots$

$$
a_{1},\left|\begin{array}{cc}
a_{1} & a_{0} \\
a_{3} & a_{2}
\end{array}\right|,\left|\begin{array}{ccc}
a_{1} & a_{0} & 0 \\
a_{3} & a_{2} & a_{1} \\
a_{5} & a_{4} & a_{3}
\end{array}\right|,\left|\begin{array}{cccc}
a_{1} & a_{0} & 0 & 0 \\
a_{3} & a_{2} & a_{1} & a_{0} \\
a_{5} & a_{4} & a_{3} & a_{2} \\
a_{7} & a_{6} & a_{5} & a_{4}
\end{array}\right|, \quad \ldots .
$$

Mit Hilfe dieses Kriteriums, für dessen Beweis wir auf die Hurwitzsche Abhandlung verweisen, kann nun der Hilfssatz 3 ohne Schwierigkeit bewiesen werden:

Man betrachte in der Tat das Polynom

$$
(x+1)^{n}=x^{n}+\left(\begin{array}{c}
n \\
1
\end{array}\right) x^{n-1}+\left(\begin{array}{l}
n \\
2
\end{array}\right) x^{n-2}+\ldots+\left(\begin{array}{l}
n \\
n
\end{array}\right)
$$

und beachte, dass dieses die (negative) Wurzel -1 als $n$-fache Wurzel hat. Daraus folgt, dass die mit Hilfe der Koeffizienten $\left(\begin{array}{l}n \\ 0\end{array}\right),\left(\begin{array}{l}n \\ 1\end{array}\right), \ldots,\left(\begin{array}{l}n \\ n\end{array}\right)$ gebildeten Determinanten $\Delta_{\lambda} \quad(\lambda=1,2, \ldots, n)$ positiv sind. Da jetzt

$$
\Delta_{n}=\left|\left(\begin{array}{c}
n \\
2 s-k-1
\end{array}\right)\right|
$$

ist, so ist $\Delta_{n}>0$ und somit $\neq 0$.

Ist nun die durch (6.9) definierte Grösse $\Delta_{n}$ ungleich Null, so müssen alle $\bar{f}((2 s-1) /(2 n)) \quad(s=1,2, \ldots, n) \quad$ verschwinden. Denn für jedes (feste) $\mu=1,2, \ldots, n$ ist das System

$$
\sum_{k=0}^{n}\left(\begin{array}{c}
n \\
2 s-k-1
\end{array}\right) \alpha_{k}=\delta_{s \mu} \quad(s=1,2, \ldots, n)
$$

(wobei $\delta_{s \mu}$ das bekannte Kronecker-Symbol bedeutet) lösbar, und man kann durch die Wahl der $\alpha_{k}\left(\alpha_{k}=\alpha_{k}(\mu)\right)$ erreichen, dass jedes $\bar{f}((2 s-1) /(2 n)) \quad(s=1,2, \ldots, n) \quad$ verschwindet. Somit ist

$$
\bar{f}\left(\frac{k}{2 n}\right)=0 \quad(k=0,1, \ldots, 2 n) .
$$


Wiederholt man dieses Verfahren $q$-mal, so erhält man die Gleichungen

$$
\bar{f}\left(\frac{k}{2^{q} n}\right)=0 \quad\left(k=0,1,2, \ldots, 2^{q} n\right) .
$$

Danach verschwindet $\bar{f}(x)$ auf $N_{0}$ und mithin (da $f$ noch stetig ist) auch auf $J=[0,1]$.

Der Beweis des Hilfssatzes 2 verläuft analog und wird hier nicht explizit durchgeführt.

7. Anwendung auf die Theorie der gewöhnlichen Differentialgleichungen. Die Anwendung der Hilfssätze 1 und 2 auf die Differentialgleichungen

$$
D^{n} f(x)=g(x, f(x)),
$$

bei geeignetem Wertevorrat von $f$ und $g$, bringt den Nachweis, dass $D^{n} f(x)$, sofern $f(x)$ eine $n$-te Ableitung $f^{(n)}(x)$ hat, gleich $f^{(n)}(x)$ ist. Dabei wird angenommen, dass $x$ in einem Intervall $J_{0}=\left[a_{0}, b_{0}\right]$ $\left(a_{0}<b_{0}\right)$ von $\boldsymbol{R}$ variiert, und dass $f$ in einem Banach-Raum $\boldsymbol{B}$ liegt. Grundlegend für nachfolgende Entwicklungen ist der Satz:

Hilfssatz 4. Hat die stetige Abbildung $f: J_{\mathbf{0}} \rightarrow \boldsymbol{R}$ eine n-te Ableitung $f^{(n)}\left(x_{0}\right)$ in einem Punkt $\left.x_{0} \in\right] a_{0}, b_{0}\left[\right.$, so existiert $D^{n} f\left(x_{0}\right)$, und es gilt,

$$
D^{n} f\left(x_{0}\right)=f^{(n)}\left(x_{0}\right) \text {. }
$$

Beweis. Für $m \geqq 0$, ganz, ist

$$
A_{m}=\sum_{k=0}^{n}(-1)^{k}\left(\begin{array}{l}
n \\
k
\end{array}\right) \frac{k^{m}}{n^{m}}=\left.\frac{1}{n^{m}} \frac{d^{m}}{d \alpha^{m}}\left(1-e^{\alpha}\right)^{n}\right|_{\alpha=0},
$$

und somit

$$
A_{m}=\left\{\begin{array}{ll}
0 & (0 \leqq m<n) \\
(-1)^{n} \frac{n !}{n^{n}} & (m=n)
\end{array} .\right.
$$

Daraus folgt für $\left.J_{x_{0}}=[a, b] \subset\right] a_{0}, b_{0}\left[\left(a<b, x_{0} \in J_{x_{0}}\right)\right.$, wegen

also

$$
(b-a)^{n} D^{n}\left(J_{x_{0}}, x^{m}\right)=\sum_{k=0}^{n}(-1)^{k}\left(\begin{array}{l}
n \\
k
\end{array}\right)\left[a+(b--a) \frac{k}{n}\right]^{m}
$$

$$
\begin{gathered}
D^{n}\left(J_{x_{0}}, x^{m}\right)=\sum_{\lambda=0}^{m}\left(\begin{array}{c}
m \\
\lambda
\end{array}\right) A_{\lambda} a^{m-\lambda}(b-a)^{\lambda-n}, \\
D^{n} x^{m}=\left(x^{m}\right)^{(n)} .
\end{gathered}
$$


Hat nun $f$ in $\left.x_{0} \in\right] a_{0}, b_{0}\left[\right.$ eine $n$-te Ableitung $f^{(n)}\left(x_{0}\right)$, so gilt nach der Taylor-Formel für alle $J_{x_{0}}$ mit hinreichend kleiner Länge $\left|J_{x_{0}}\right|$

$$
f(x)=P_{n-1}(x)+\left(x-x_{0}\right)^{n}\left\{\frac{f^{(n)}\left(x_{0}\right)}{n !}+o(1)\right\},
$$

wobei $P_{n-1}(x)$ ein Polynom vom Grade $\leqq n-1$ ist und $|o(1)|$ mit $\left|J_{x_{0}}\right| \rightarrow 0$ gegen Null strebt. Das liefert in Verbindung mit (7.3) die Gleichung (7.2).

Der Hilfssatz 4 gilt, in ähnlicher Form, auch dann, wenn $f$ eine stetige Abbildung von $I_{\mathbf{0}} \subset \boldsymbol{B}_{\mathbf{0}}$ in einen Banach-Raum $\boldsymbol{B}$ liefert und $f=$ $f\left((1-\lambda) a_{0}+\lambda b_{0}\right)$ als Funktion von $\lambda$ in einem Punkt $\lambda_{0}$ von $(0,1)$ eine $n$-te Derivierte in gewöhnlichem Sinne besitzt.

Die Übertragung der Hauptergebnisse dieser Arbeit ${ }^{8}$ ) auf partielle Differentialgleichungen von der Form

$$
\frac{\partial^{m+n} V}{\partial x^{m} \partial y^{n}}=f(x, y ; V)
$$

(und entsprechend für mehrere Veränderliche) geschieht durch Heranziehung der Entwicklungskoeffizienten von $(1-x)^{m}(1-y)^{n}$ und Anwendung eines entsprechend verallgemeinerten Goursatschen Zerstückelungsverfahrens. Die ausführliche Darstellung der gewonnenen Zusammenhänge soll später gegeben werden.

Freie Universität Berlin

Deutschland

8) Auf eine Reihe von Ergebnissen "in der Norm», deren Existenz ich durch Herrn Professor P. L. Butzer (Aachen) während einer Tagung über Funktionalanalysis im August 1965 in Oberwolfach erfahren habe, will ich hier um so weniger eingehen, als dadurch Hauptziel der vorliegenden Arbeit, die zunächst ohne jeglichen Zusammenhang mit vorhandener Literatur geschrieben wurde, nicht allzu sehr berührt wird. 


\section{Literatur}

[1] Cantor, G.: Beweis, dass eine für jeden reellen Wert von $x$ durch eine trigonometrische Reihe gegebene Funktion $f(x)$ sich nur auf eine einzize Weise in dieser Form darstellen lässt. - Gesammelte Abhandlungen, Verlag von Julius Springer, Berlin, 1932, S. 80-83.

[2] Hagen, J. G.: Synopsis der höheren Mathematik. I. - Dames Verlag, Berlin, 1891.

[3] Hille, E., und R. S. Phillips: Functional analysis and semi-groups. - Colloquium Publications 31, American Mathematical Society, Providence (R. I.), 1957. [Zweite revidierte Auflage des gleichnamigen Buches von E. HiLle.]

[4] Hurwitz, A.: Ueber die Bedingungen, unter welchen eine Gleichung nur Wurzeln mit negativen reellen Theilen besitzt. - Math. Ann. 46, 1895, S. 273-284.

[5] JAmes, R. D.: A generalized integral. II. - Canad. J. Math. 2, 1950, S. 297-306.

[6] Jeffery, R. L.: Trigonometric series. - Canadian Mathematical Congress Lecture Series 2, University of Toronto Press, Toronto, 1956.

[7] Lorentz, G. G.: Bernstein polynomials. - Mathematical Expositions 8, University of Toronto Press, Toronto, 1953.

[8] Netro, E.: Lehrbuch der Combinatorik. - Druck und Verlag von B. G. Teubner, Leipzig/Berlin, 1927.

[9] Riemann, B.: Ueber die Darstellbarkeit einer Funktion durch eine trigonometrische Reihe. - Gesammelte mathematische Werke und wissenschaftlicher Nachlass, Zweite Auflage, Druck und Verlag von B. G. Teubner, Leipzig, 1892, S. 227-265.

[10] Schwartz, H. A.: Beweis eines für die Theorie der trigonometrischen Reihen in Betracht kommenden Hülfssatzes. - Gesammelte mathematische Abhandlungen. II, Verlag von Julius Springer, Berlin, 1890, S. 341-343.

[11] Weber, H.: Lehrbuch der Algebra. I. - Zweite Auflage, Druck und Verlag von Friedrich Vieweg und Sohn, Braunschweig, 1898. 